\title{
Reducir la exclusión social des las niñas
}

Kelly Hallman

Population Council

Eva Roca

Population Council

Follow this and additional works at: https://knowledgecommons.popcouncil.org/departments_sbsr-pgy

Part of the Demography, Population, and Ecology Commons, Family, Life Course, and Society

Commons, Gender and Sexuality Commons, International Public Health Commons, and the Medicine and Health Commons

How does access to this work benefit you? Let us know!

\section{Recommended Citation}

Hallman, Kelly and Eva Roca. 2007. "Reducir la exclusión social des las niñas," Promoción de transiciones a la vida adulta sanas, seguras y productivas Resumen no. 27. New York: Population Council. 


\section{Reducir la exclusión social des las niñas}

\section{Preparado por Kelly Hallman y Eva Roca}

E $\mathrm{n}$ todo el mundo, las niñas son sistemáticamente excluidas de la participación en la vida social, económica y política. La ausencia de niñas en estos terrenos tiene consecuencias, no sólo para las mismas jóvenes sino también para la sociedad en su totalidad, ya que se exacerba la pobreza y se perpetúan las disparidades de salud, educación y éxito económico. A nivel internacional, esta marginación dificulta o hace imposible que algunos países logren objetivos en toda la sociedad, como los Objetivos de Desarrollo del Milenio, considerados por las Naciones Unidas como parámetros para reducir la pobreza.

La exclusión social de las mujeres comienza a temprana edad y es especialmente notoria en las transiciones de la vida como la pubertad y el matrimonio. La exclusión también resulta evidente en muchos de los obstáculos con que tropiezan las niñas durante su transición a la adultez. Las niñas adolescentes se enfrentan a obstáculos para ingresar y permanecer en la escuela, encontrar trabajo, hacer amistades, aprender aptitudes para la vida, acceder a los servicios de salud y participar en la vida cívica. En algunas partes de Asia, el aborto selectivo a causa del sexo y el infanticidio femenino reduce las posibilidades de las niñas a comenzar siquiera a vivir (Drèze y Sen, 1989).

Desde hace algunos años, el Population Council ha estado estudiando las causas y efectos de la exclusión social de las niñas en los países en desarrollo, mediante proyectos en Asia, África y Latinoamérica.

\section{¿Cuáles niñas son las más excluidas?}

La exclusión de las niñas es más pronunciada en los países extremadamente pobres y aquéllos donde las mujeres han sido marginadas históricamente (como por ejemplo en el Oriente Próximo). En numerosos países en desarrollo, la exclusión social de las mujeres se debe a la interacción entre los géneros, cultura y pobreza. Algunos cálculos recientes indican que tres cuartas partes de los 60 millones de niñas entre 6 y 11 años de edad que no asisten a la escuela pertenecen a minorías étnicas, religiosas, lingüísticas, raciales u otras minorías (Lewis y Lockheed, 2006). Las niñas pertenecientes a estas minorías sufren de manera desproporcionada, comparadas con sus pares varones y con las

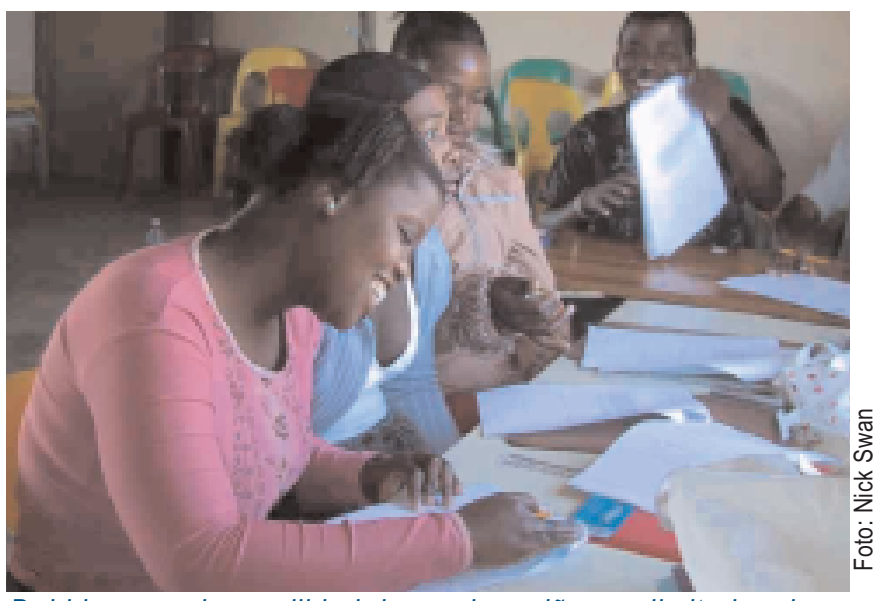

Debido a que la movilidad de muchas niñas es limitada, el requerimiento principal para su inclusión social es un espacio seguro y de apoyo donde puedan interactuar con compañeros de su misma edad y mentores.

niñas de la población dominante. Por ejemplo, entre la población de siete años de edad en Guatemala en el año 2000, sólo el 54\% de las niñas indígenas estaban inscritas en la escuela, comparado con el $71 \%$ de niños indígenas y el $75 \%$ de niños no indígenas. Entre las niñas indígenas extremadamente pobres, la proporción inscrita era del 43\% (Hallman y otros, 2007b).

En algunos casos, los obstáculos provocados por el género, la pobreza y el origen étnico son exacerbados por la exclusión geográfica: ser del "lado equivocado del pueblo" o residir en una comunidad remota. Por lo general, las niñas tienen una movilidad limitada con respecto a sus pares varones, de modo que los servicios y las oportunidades que no se encuentran en el vecindario cercano de una niña pueden estar fuera de su alcance. También es posible que las niñas carezcan de los medios financieros para viajar con seguridad y acceder a los empleos 0 a las oportunidades educativas.

\section{La exclusión social de las niñas constituye una barrera para el desarrollo}

La exclusión social de las niñas perpetúa la pobreza en los niveles individual y doméstico, pues se les niega acceso a 
educación, servicios, recursos, toma de decisiones y mercados laborales. Las expectativas y las experiencias reales de exclusión y discriminación pueden causar sentimientos de impotencia entre las personas excluidas, lo que puede dar como resultado una baja autoestima y una disminución de aspiraciones para el futuro. La investigación ha demostrado que esos sentimientos pueden causar un nivel de logros inferior entre los miembros de los grupos excluidos. Por ejemplo, en un estudio realizado en India, se descubrió que el desempeño de los jóvenes provenientes de castas bajas y castas altas era igualmente bueno en aquellos exámenes donde no se revelaba información sobre su casta. Sin embargo, cuando las castas se anunciaron públicamente, el desempeño de los niños de casta inferior fue peor que el de sus pares de casta superior (DFID 2005). Si los padres suponen que sus hijas serán discriminadas en el mercado laboral, es posible que elijan no invertir en su educación. Como observó una joven participante en un proyecto del Council en Guatemala (Colom y otros, 2004; Dries-Daffner y otros, 2007):

A veces no quieren pagar nuestra educación sólo porque somos mujeres, y dicen que sólo los niños deberían [estudiar], porque son más inteligentes. Algunas personas le dicen a mi padre: "No pagues sus estudios porque ella no hará lo que tú quieres, sólo malgastarás tu dinero; ella se casará y no terminará la escuela, es mejor que no la envíes a la escuela."

En zonas gravemente afectadas por el VIH y el SIDA, las expectativas de un período de vida corto puede servir para disminuir el valor del futuro y reducir las inversiones en el capital humano. Es posible que ahorrar para el futuro o privarse de realizar actividades hoy, para estar sanas y bien educadas el día de mañana, no parezca sensato para las jóvenes amenazadas por el VIH/SIDA. Por otra parte, los resultados de Sudáfrica indican que las jóvenes con actitudes más orientadas hacia el futuro (por ejemplo, aquéllas que tienen objetivos financieros y están ahorrando) tienen más conocimientos sobre VIH y tienen mayores posibilidades de poner en práctica conductas de prevención de VIH (Hallman y otros, 2007a).

La exclusión social de las niñas tiene consecuencias a nivel nacional. Aun cuando las economías nacionales crecen, los grupos excluidos quedan rezagados. El aislamiento social y la relativa privación económica están asociados a una salud mental más pobre, especialmente entre mujeres, y pueden reducir aún más la capacidad de las personas excluidas para ser miembros productivos de la sociedad (Patel y Kleinman, 2003). A medida que se incrementa la brecha entre pobres y no pobres, la pobreza se torna más profunda e inextricable.

\section{Causas subyacentes de la exclusión de las niñas}

Ya sea intencional (debido a la tradición o a la discriminación) o involuntario (por el deseo de proteger a las niñas de daños o amenazas a su castidad), el resultado es el mismo: desde todo punto de vista, la vida de las niñas está más limitada que la de los niños.

A partir de una edad temprana, las niñas que residen en las mismas comunidades y hogares que los niños son más excluidas de las inversiones y las oportunidades. En muchos entornos, la pubertad es la época en que las expectativas de los roles de género comienzan a diferenciarse y a intensificarse. Los niños experimentan más libertad para tomar decisiones y más movilidad, mientras que el movimiento de las niñas fuera de la esfera doméstica está cada vez más limitado (Mensch y otros, 2003), a menudo porque los padres temen a la atención masculina, la tentación de las actividades no autorizadas y el daño potencial a la reputación de sus hijas (Brady, 2003; Colom y otros, 2004). El hecho de que los niños reciban la mayor parte de la atención de la casa, de los recursos y de la libertad se relaciona con la percepción de que los niños contribuyen más a la economía de la casa, y de que los padres pueden depender de ellos para su seguridad durante la vejez. Por el contario, se considera que las niñas contribuyen menos en cuanto a trabajo y capacidad para generar ingresos, y en algunos contextos, incluso son vistas como una carga para las finanzas de la casa, debido a los costos asociados a su matrimonio. La inversión adicional en los niños se manifiesta en sus logros en comparación con los de las niñas en cuanto a educación, alfabetismo y participación en la fuerza laboral.

La investigación realizada por el Population Council brinda evidencia proveniente de un número de entornos, según la cual las niñas adolescentes carecen de apoyo y de redes sociales que las protejan. En Allahabad, India, el 93\% de los niños, pero sólo el $22 \%$ de las niñas, informaron poder viajar solos para visitar a un pariente (Sebastian y otros, 2004). En los barrios bajos urbanos de Nairobi, las dos terceras partes de los niños, en comparación con sólo una tercera parte de las niñas, informaron tener un sitio seguro donde reunirse con amigos del mismo sexo (Erulkar y Chong, 2005). Entre los adolescentes de Addis Ababa, Etiopía, los varones informaron tener un promedio de 4.7 amigos, en comparación con 2.7 amigas informadas por las niñas (Erulkar y otros, 2004a). En KwaZulu-Natal, Sudáfrica, el 76\% de los niños, comparado con el $48 \%$ de las niñas, informó tener muchos amigos. Las niñas más pobres fueron las que informaron tener menos amigas, e incluso las niñas pertenecientes al quintil superior de riqueza informaron tener menos amigas que los niños del quintil más pobre (Hallman y Diers, 2004). En Etiopía y Sudáfrica, fue mucho más probable que las niñas se sintieran inseguras en su vecindario y que hubieran experimentado acoso; también fue menos probable que informaran tener fuentes de apoyo en épocas de crisis (un lugar donde quedarse o alguien a quien pedirle dinero prestado). En las áreas rurales tanto de Guatemala como de Etiopía, los varones tuvieron mayores posibilidades que las mujeres de participar en eventos sociales (Colom y otros, 2004; Erulkar y otros, 2004b). En las áreas rurales de Egipto Superior, la única salida social no familiar para las niñas es asistir a la escuela (Brady y otros, 2007). 
Para una gran cantidad de niñas, el matrimonio es el único medio socialmente aceptable para salir de un hogar natal pobre y excesivamente protector, y muchas niñas esperan con ansias el momento de casarse, pues creen que de este modo expandirán su horizonte social (Colom y otros, 2004; Brady y otros, 2007). Sin embargo, esta expectativa muchas veces no se materializa. La investigación del Council indica que las jóvenes casadas tienen redes de pares más limitadas (Amin, Mahmud y Huq, 2002), menos movilidad social y libertad (Erulkar y otros, 2004b; Santhya y Jejeebhoy, 2003), un acceso más limitado a los medios de comunicación y a otras fuentes de información (Erulkar y otros, 2004b; Amin, Mahmud, y Huq, 2002), y logros educativos más bajos (Mensch 2005) que sus compañeras solteras de la misma edad. Comparadas con las mujeres que se casan más tarde, las adolescentes casadas tienen esposos mucho mayores que ellas (Clark, Bruce y Dude, 2006; Mensch, Bruce y Greene, 1998). También tienen menos libertad de movimiento (Amin, Mahmud y Huq, 2002), menos autonomía y poder de decisión en el hogar y en las decisiones reproductivas (Santhya y Jejeebhoy, 2003) y, en algunos entornos, un riesgo mayor de violencia de género (Kishor y Johnson, 2004), infecciones transmitidas sexualmente e infección por VIH (Clark, Bruce y Dude, 2006).

\section{Estrategias promisorias para incluir a las niñas adoles- centes}

Aunque todavía queda mucho trabajo por hacer, existen varios enfoques que están probando ser beneficiosos para las jóvenes. Debido a que la movilidad de muchas niñas es restringida, especialmente cerca de la pubertad, el requerimiento principal para su inclusión social es un espacio seguro y de apoyo donde poder interactuar con sus pares y mentores, fortalecer sus redes sociales y disfrutar de libertad de expresión y de movimiento. Los espacios seguros para las niñas pueden servir como lugares para varios servicios beneficiosos, entre ellos educación financiera y comercial, intervenciones relacionadas con temas de la salud, asesoramiento para tener más confianza en sí mismas, y desarrollo de habilidades para la negociación sexual. Los espacios seguros también actúan como base para construir la capacidad de organización y movilización de las niñas.

A fin de participar plenamente en la vida cívica, las jóvenes necesitan tener acceso a documentación, como por ejemplo documento de identidad y otros documentos del gobierno. También requieren acceso a servicios de salud y otros servicios orientados a la juventud. Los resultados de varios estudios llevados a cabo por el Council revelan que los adolescentes más aislados socialmente son los que tienen menos probabilidades de estar en contacto con centros juveniles, clínicas aptas para adolescentes y programas de educación a través de pares (Lardoux y Jones, 2006). Irónicamente, las mismas adolescentes que más necesitan estos servicios -las jóvenes pobres, casadas, que viven en zonas rurales, que no asisten a la escuela y/o viven sin uno 0 ambos padres- son las que menos acceden a estos servicios (Bruce y otros, 2006; Lardoux y Jones, 2006). Es necesario esforzarse para aumentar los servicios disponibles y asegurar el acceso de las niñas a esos servicios.

\section{Recomendaciones del programa}

Deben diseñarse programas de reducción de la pobreza y otros programas para llegar con eficacia a estas niñas excluidas y brindarles información y servicios. Para ser exitosos, los programas deben aprender del contexto local y adaptarse a él. Además, los programas pueden ayudar a potenciar a las niñas si se modifican las actitudes de la comunidad. Si los padres y otros tutores reconocen la importancia de incluir a las niñas, es más probable que éstas pasen a formar parte visible de su comunidad. La tarea del Council en Egipto (Brady y otros, 2007) demostró que es posible modificar las actitudes de la comunidad con respecto a las niñas.

\section{Brechas en la investigación}

Se necesitan más investigaciones en todo el mundo para aprender más sobre qué grupos de niñas están excluidas, con el objetivo de examinar los efectos del aislamiento en sus vidas, y para desarrollar enfoques específicos del contexto para satisfacer las necesidades de las niñas. Al separar los datos existentes (como por ejemplo las Encuestas demográficas y de salud, así como otros datos de todo el país), es posible que los investigadores comprendan mejor las tendencias que se esconden detrás de los promedios. Debe analizarse en profundidad la relación que existe entre la exclusión social de las niñas y sus derechos. Asimismo, los investigadores deberían explorar los efectos negativos de la exclusión social sobre la reducción de la pobreza y el logro de los Objetivos de Desarrollo del Milenio, así como otras medidas de desarrollo humano. En definitiva, los derechos de las niñas son derechos humanos. Las niñas aisladas socialmente constituyen un grupo que no podemos abandonar.

\section{Referencias y publicaciones relacionadas}

Amin, Sajeda, Simeem Mahmud, and Lopita Huq. 2002. "Baseline survey report on rural adolescents in Bangladesh." Dhaka: Ministry of Women's Affairs, Government of Bangladesh.

Brady, Martha. 2003. "Safe spaces for adolescent girls," in Adolescent and Youth Sexual and Reproductive Health: Charting Directions for a Second Generation of Programming (background documents). New York: UNFPA, pp. 155-176.

Brady, Martha, Ragui Assaad, Barbara Ibrahim, Abeer Salem, Rania Salem, and Nadia Zibani. 2007. Providing New Opportunities to Adolescent Girls in Socially Conservative Settings: The Ishraq Program in Rural Upper Egypt. New York: Population Council.

Bruce, Judith, Nicole Haberland, Erica Chong, Monica Grant, and Amy Joyce. 2006. "The girls left behind: The failed reach of current schooling, child health, youth-serving, and livelihoods programs for girls living in the path of HIV," Policy paper. New York: Population Council. 
Clark, Shelley, Judith Bruce, and Annie Dude. 2006. "Protecting young women from HIVIAIDS: The case against child and adolescent marriage," International Family Planning Perspectives 32(2): 79-88.

Colom, Alejandra, Marta Julia Ruiz, Jennifer Catino, Kelly Hallman, Sara Peracca, and Kristen M. Shellenberg. 2004. "Voices of vulnerable and underserved adolescents in Guatemala." Guatemala City: Population Council.

DFID. 2005. "Reducing poverty by tackling social exclusion: A DFID policy paper." London: DFID.

Drèze, Jean and Amartya Sen. 1989. Hunger and Public Action. Oxford: Clarendon Press.

Dries-Daffner, Ingrid, Kelly Hallman, Jennifer Catino, and Karla Berdichevsky. 2007. "Guatemala," in International Encyclopedia of Adolescence, 2 vols., ed. Jeffrey J. Arnett. New York and London: Routledge.

Erulkar, Annabel and Erica Chong. 2005. "Evaluation of a savings and micro-credit program for vulnerable young women in Nairobi." Nairobi: Population Council.

Erulkar, Annabel S., Tekle-Ab Mekbib, Negussie Simie, and Tsehai Gulema. 2004a. "Adolescent life in low income and slum areas of Addis Ababa, Ethiopia." New York: Population Council.

Erulkar, Annabel S., Tekle-Ab Mekbib, Negussie Simie, and Tsehai Gulema. 2004b. "The experience of adolescence in rural Amhara Region, Ethiopia". New York: Population Council.

Erulkar, Annabel S., Tekle-Ab Mekbib, Negussie Simie, and Tsehai Gulema. 2006. "Migration and vulnerability among adolescents in slum areas of Addis Ababa, Ethiopia." Journal of Youth Studies 9(3): 361-374.

Hall, Gillette and Harry Anthony Patrinos. 2004. Indigenous Peoples, Poverty and Human Development in Latin America. New York: Palgrave Macmillan.

Hallman, Kelly and Judith Diers. 2004. "Social isolation and economic vulnerability: Adolescent HIV and pregnancy risk factors in South Africa," presentation at the Annual Meeting of the Population Association of America, April, Boston, MA.

Hallman, Kelly, Kasthuri Govender, Emmanual Mbatha, Jill Walsh, Rob Pattman, and Deevia Bhana. 2007a. "Social capital, socioeconomic aspirations, and HIV risk behaviors among poor South African youth," poster presentation at the Third South African AIDS Conference, June, Durban, South Africa.

Hallman, Kelly and Sara Peracca, with Jennifer Catino and Marta Julia Ruiz. 2007b (forthcoming). "A four-fold disadvantage: The effects of ethnicity, gender, poverty, and geography on education in Guatemala," in Maureen Lewis and Marlaine Lockheed (eds.), Exclusion, Gender and Education: Case Studies from the Developing World. Washington, DC: Center for Global Development.

Kishor, Sunita and Kiersten Johnson. 2004. Profiling Domestic Violence: A Multi-Country Study. Calverton, MD: ORC Macro.

Lardoux, Solene and Heidi Jones. 2006. "Capacity building for adolescent program and policy development in West Africa: Interim narrative report." New York: Population Council.
Lewis, Maureen A. and Marlaine E. Lockheed. 2006. Inexcusable Absence: Why 60 Million Girls Still Aren't in School and What to Do About It. Washington, DC: Center for Global Development.

Lloyd, Cynthia B., Cem Mete, and Monica Grant. 2007 (forthcoming). "Rural girls in Pakistan: Constraints of policy and culture," in Maureen Lewis and Marlaine Lockheed (eds.), Exclusion, Gender and Schooling: Case Studies from the Developing World. Washington, DC: Center for Global Development.

Mensch, Barbara S. 2005. "The transition to marriage," in Cynthia B. Lloyd (ed.), Growing Up Global: The Changing Transitions to Adulthood in Developing Countries. Washington, DC: National Academies Press, pp. 416-505.

Mensch, Barbara S., Judith Bruce, and Margaret E. Greene. 1998. The Uncharted Passage: Girls' Adolescence in the Developing World. New York: Population Council.

Mensch, Barbara S., Barbara L. Ibrahim, Susan M. Lee, and Omaima El-Gibaly. 2003. "Gender-role attitudes among Egyptian adolescents," Studies in Family Planning 34(1): 8-18.

Patel, Vikram and Arthur Kleinman. 2003. "Poverty and common mental disorders in developing countries." Bulletin of the World Health Organization 81(8): 609-615.

Population Council. Various years. "Facts about adolescents from the Demographic and Health Survey: Statistical tables for program planning" (various countries, for full list see: $<$ <ttp://www.popcouncil.org/gfd/gfdcountries.html>. New York: Population Council.

Santhya, K.G. and Shireen J. Jejeebhoy. 2003. "Sexual and reproductive health needs of married adolescent girls," Economic and Political Weekly 38(41): 4370-4377.

Sebastian, Mary P., Monica Grant, and Barbara Mensch. 2004. "Integrating adolescent livelihood activities within a reproductive health programme for urban slum dwellers in India." New Delhi: Population Council.

Shisana, O., T. Rehle, L.C. Simbayi, W. Parker, K. Zuma, A. Bhana, C. Connolloy, S. Jooste, and V. Pillay. 2005. South African National HIV Prevalence, HIV Incidence, Behavior and Communication Survey. Capetown: HSRC Press.

UNDP. 1995. Human Development Report 1995: Gender and Development. New York: Oxford University Press.

\section{Donantes}

Department for International Development (DFID), The Dickler Family Foundation, EMPower-The Emerging Markets Foundation, Focus on Young Adults, The Ford Foundation, Bill \& Melinda Gates Foundation, The William H. Kaufman Charitable Foundation, MEASURE Evaluation, The Andrew W. Mellon Foundation, The Rockefeller Foundation, The Summit Foundation, Turner Foundation, United Nations Children's Fund (UNICEF), United States Agency for International Development (USAID)

Para obtener más información o copias de los resúmenes de esta serie, escriba a publications@popcouncil.org Para consultar sobre recursos adicionales, visite www.popcouncil.org/pgy

Population Council

One Dag Hammarskjold Plaza

Nueva York, NY 10017 E.U.A.

(c) 2007 The Population Council, Inc. 